\title{
Hydrodynamic interaction of bubbles rising side-by-side in viscous liquids
}

\author{
G Kong $^{1} \cdot$ H. Mirsandi ${ }^{1}$ K. A. Buist ${ }^{1}$ - E. A. J. F. Peters ${ }^{1}$ (I) $\cdot$ M. W. Baltussen ${ }^{1} \cdot$ J. A. M. Kuipers ${ }^{1}$
}

Received: 20 May 2019 / Revised: 5 August 2019 / Accepted: 25 August 2019 / Published online: 17 September 2019

(c) The Author(s) 2019

\begin{abstract}
Detailed experiments are conducted to study hydrodynamic effects of two simultaneously released bubbles rising in viscous liquids. Different types of interactions are observed as a function of the liquid viscosities, leading to different bubble shapes, ranging from rigid spheres and spheroids to deformable spheroids. Bubble velocities are obtained by an automated smooth spline technique, which allows for an accurate calculation of the lift and drag forces. The results obtained for spherical bubbles are in agreement with predictions of Legendre et al. (J Fluid Mech 497:133-166, 2003). The observations of deformed bubbles show that a very small equilibrium distance can be established due to the induced torque arising from the deformation. In terms of the lateral interaction, different separation distances can be observed depending on the initial distance. For deformable bubbles, the results are limited to a qualitative analysis due to limitations of the processing technique to handle strong shape irregularities. Nevertheless, the observations reveal that the deformation plays an important role with respect to bubble interactions and path instability of which the latter can be triggered by the presence of other bubbles.
\end{abstract}

\section{Graphic abstract}
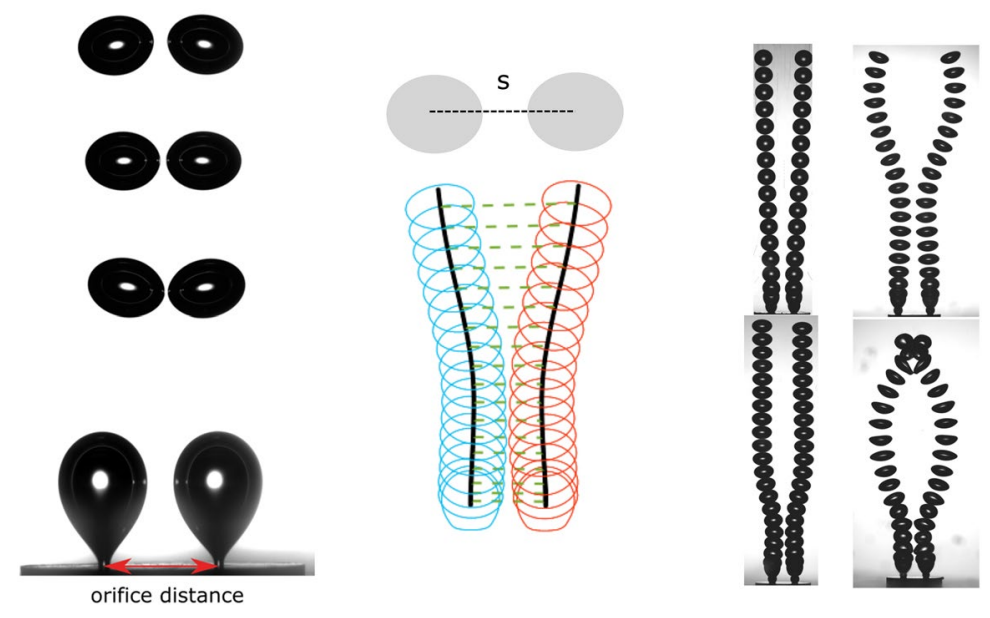

\section{List of symbols}

\section{Dimensionless numbers}

$\mathrm{Ca} \quad \mathrm{Ca}=\mu U / \sigma$. Capillary number

Eo $\rho_{l} g D_{\text {eq }}^{2} / \sigma$. Eötvös number

\section{E. A. J. F. Peters}

e.a.j.f.peters@tue.nl

1 Department of Chemical Engineering and Chemistry, Eindhoven University of Technology, P.O. Box 513, 5600 MB Eindhoven, The Netherlands

\footnotetext{
Oh $\mu /\left(\rho_{l} \sigma D_{\text {eq }} / 2\right)^{1 / 2}$. Ohnesorge number

$\operatorname{Re} \rho_{l} U D / \mu$. Reynolds number

We $\rho_{l} U^{2} D / \sigma$. Weber number

Mo $\quad M o=g \mu_{l}^{4} /\left(\rho \sigma^{3}\right)$. Morton number

$\chi \quad R_{1} / R_{2}$. Bubble aspect ration

\section{Greek symbols}

$\rho \quad$ Density

$\epsilon \quad$ Measurement error

$\theta \quad$ Pitch angle
} 
$\boldsymbol{\Omega}$ Angular velocity

$\Gamma$ Torque

$\sigma \quad$ Surface tension

\section{Latin symbols}

a Acceleration

A Added mass tensor

A Surface area

$C_{\mathrm{D}} \quad$ Drag coefficient

$C_{1} \quad$ Lift coefficient

D Rotational added mass tensor

$D_{\text {eq }}$ Equivalent diameter

E Energy

F Force

$f_{\text {req }}$ Recording frequency

$R_{1} \quad$ Semi-major axis

$R_{2} \quad$ Semi-minor axis

$s \quad$ Distance between two centres

$S \quad$ Dimensionless distance between two centres

$t \quad$ Time

$U$ Bubble velocity

$V \quad$ Bubble volume

\section{Introduction}

Bubbles are often encountered in industrial, biochemical, and environmental processes. In these processes, bubbles interact with the liquid phase and other bubbles and significant experimental and computational efforts have been made to obtain closures for drag and lift forces. However, the behaviour of rising gas bubbles (isolated or in swarms) is very complex and even the simplest case such as the rise of a single deformable air bubble is quite complex. Some of the behaviour is still under investigation and not completely understood, such as the path instability of rising bubbles (Magnaudet and Eames 2000; Ern et al. 2012). In industrial applications, bubbles in dense swarms are encountered revealing complex interactions and additional effects of effective drag and lift and earlier onset of path instability. Thus, the behaviour of multiple bubbles has attracted more and more attention due to its practical significance. For instance, the disturbance from a neighbouring bubble plays a significant role in bubble rising behaviour such as coalescence and clustering. Coalescence is usually unfavourable in industrial applications, because it decreases the overall surface area and hence deteriorates mass and heat transfer rates. In addition, coalesced bubbles alter the flow due to stronger deformation and a larger moving interface. The enhanced interaction of multiple bubbles often leads to local clustering, which consequently alters the flow field and the mass and heat transfer characteristics. Despite extensive research efforts, a lot of open questions remain.
To understand the physics underlying bubble swarms, a lot of effort has been made as well. Analytical studies (Van Wijngaarden and Jeffrey 1976) and simulations based on irrotational flow (Sangani and Didwania 1993; Smereka 1993; Yurkovetsky and Brady 1996) have revealed that bubbles aggregate in the horizontal plane due to the interaction. However, experiments show that low volume fraction bubbly flows tend to be homogeneously dispersed. Discrepancy between simulation and experiment has been attributed to the improper assumption that bubbles bounce elastically. Alternatively, taking deformability of the bubbles into account, front tracking (FT) simulations (Bunner and Tryggvason 2003; Esmaeeli and Tryggvason 2005) have revealed that spherical bubbles tend to align horizontally, whereas deformable bubbles tend to uniformly distribute over the volume under consideration. Apart from the clustering, Cartellier and Rivière (2001) and Risso and Ellingsen (2002) have shown that wake interaction among multiple bubbles causes bubble-induced turbulence, which has a strong impact on the efficiency of heat and mass transfer. Studies of Roghair et al. (2011a, b, 2013a, b) have resulted in several correlations quantifying the swarm effect on the effective drag coefficient. Recently, Loisy et al. (2017) studied bubbles rising in configurations of ordered and freely rising bubble arrays. In addition, the velocity fluctuation of rising bubbles due to the swarm effect was studied experimentally in spite of the experimental limitation at high gas volume fraction (Martínez-Mercado et al. 2007; Riboux et al. 2010; Colombet et al. 2015). Moreover, bubbles rising in a thin gap were studied as a model system (Bouche et al. 2012, 2014; Roig et al. 2012), in which the turbulence is suppressed.

From the studies reported in literature, it is clear that the interaction between neighbouring bubbles alters the bubbly flow globally. However, bubble-pair interaction has drawn a lot of attention due to its evident relevance to understand more complex systems. The interaction of either inline bubble pairs or side-by-side pairs has been frequently studied. Harper (1970) calculated the interaction between a pair of spherical inline bubbles and found that an equilibrium distance between the bubbles exists based on potential theory. Yuan and Prosperetti (1994) numerically investigated a similar configuration and confirm the presence of the equilibrium distance indicating the importance of the viscous force. Experimental research by Katz and Meneveau (1996), however, was not in agreement with these predictions, showing that pairs of small bubbles tend to collide and coalesce instead. Experiments by Sanada et al. (2005) have revealed the presence of an equilibrium distance. However, in their study, the equilibrium distance was not stable and larger than predicted by the previous studies. Finally, recent 3D DNS simulations by Gumulya et al. (2017) again revealed the existence of an equilibrium distance, leaving the discussion open to debate. 
The simultaneous release of two bubbles rising side-byside has been studied by a large number of researchers (Kok 1993; Van Wijngaarden 1993, 2005; Duineveld 1995, 1998; Legendre et al. 2003; Sone et al. 2008; Sanada et al. 2009; Hallez and Legendre 2011; Zhang et al. 2019). Van Wijngaarden (1993) calculated the interaction of a pair of bubbles using a two-bubble probability density function and compared his results to the experimental data of Kok (1993). A numerical analysis conducted by Legendre et al. (2003) indicates that the approach or separation of the bubbles depends on the Reynolds number. At lower Re, the bubble pair separates due to domination of vorticity, and at higher $R e$, they approach each other due to a thinning boundary layer and dominant pressure effect. Hallez and Legendre (2011) suggested that the interaction of a pair of spherical bubbles consists of the potential effect, a viscous correction and a significant wake effect on drag and transverse forces. These results suggest that the most stable position for a pair of bubbles is a horizontal alignment, because the inline alignment causes a negative torque. They also suggested that the effects of potential and wake ejection tend to form horizontal clusters, but could be neutralised by the agitation effect induced by neighbouring bubbles. Tripathi et al. (2017) employed 3D DNS simulation for two initially spherical bubbles rising side-by-side and found that the bubble interaction is linked to strong interacting vortices. Recently, Zhang et al. (2019) studied the evolution of the vortex associated with the interaction of bubbles rising sideby-side by the fully three-dimensional DNS simulations. The experimental studies of Duineveld $(1995,1998)$ and Sanada et al. (2009) focused on collision of bubbles and indicated that the wakes of approaching bubbles play a key role in determining the subsequent bouncing/coalescence. They both attributed the separation of the bubble pair to the shedding vortices and indicated that this could be linked to the path instability of a single bubble.
Apart from studies focusing on bouncing and coalescence, the only experimental study reported in the literature is due to Kok (1993). Unfortunately, due to experimental limitations, trajectories were the only obtainable quantitative data. The opposing findings reported in the literature and the advancement of experimental techniques prompted us to undertake the present study. Moreover, to the best of our knowledge, the simulation study of Tripathi et al. (2017) and Zhang et al. (2019) is the only work addressing the interaction between deformed bubbles. In the present study, a spherical bubble pair and a deformed bubble pair are experimentally studied to investigate their hydrodynamic interaction. This paper is organised as follows: the experimental setup and processing procedures will be presented in Sect. 2. Results and discussion will be reported in Sect. 3. The conclusions will be presented in Sect. 4 .

\section{Setup and processing procedures}

\subsection{Experimental setup and liquid properties}

The measurement system incorporates a high-speed camera (PCO, dimax HD+) mounted with a Nikon lens, an LED lamp with a diffuser plate as the illumination source and a glass column sized $100 \times 100 \times 500 \mathrm{~mm}$ (see Fig. 1). The bubble pair was generated at the nozzle with two orifices submerged at the bottom of the column. The nozzle was made of stainless steel and the surface was polished to minimise the effect of roughness. The diameter of the orifices was $1 \mathrm{~mm}$. The distance between the orifices was varied from $4 \mathrm{~mm}, 6 \mathrm{~mm}$ to $7 \mathrm{~mm}$. The orifices were connected to a two-channel syringe pump (KD Scientific LEGATO 100) mounted with two gas-tight syringes (Hamilton).

For the experiments, water-glycerol mixtures were used to vary the liquid viscosity (see Table 1) and as a
Fig. 1 Features of the four possible modes corresponding to $\mathbf{a}$ setup, $\mathbf{b}$ bubble pair generated at two orifices and rising side-byside (scales are different), and c centres of mass and distances of two centres; the dimensionless distance of two centres is defined as $S=2 s / D_{\text {eq }}$ (a)

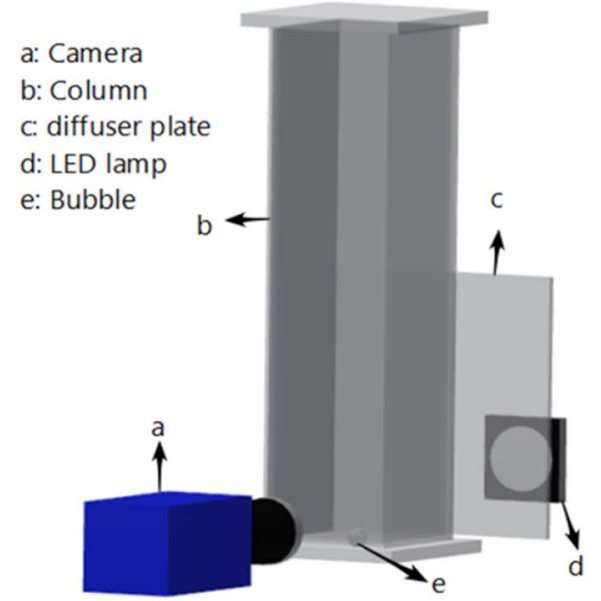

(b)

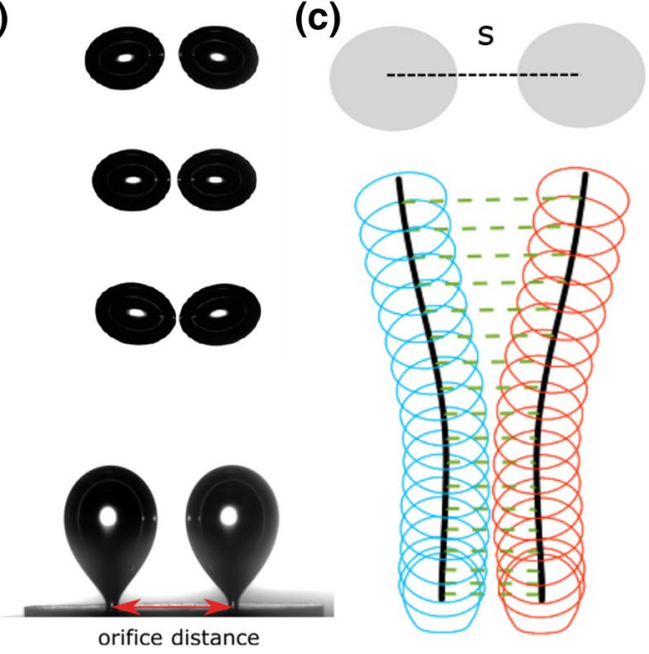


Table 1 Physical properties of liquid used in the experiments

\begin{tabular}{llll}
\hline Liquid & $\rho\left(\mathrm{kg} \mathrm{m}^{-3}\right)$ & $\mu\left(\mathrm{kg} \mathrm{m}^{-1} \mathrm{~s}^{-1}\right)$ & $\sigma\left(\mathrm{Nm}^{-1}\right)$ \\
\hline $80 \mathrm{wt} \%$ glycerol & 1208.5 & $60.1 \times 10^{-3}$ & $6.5 \times 10^{-2}$ \\
$60 \mathrm{wt} \%$ glycerol & 1153.8 & $10.8 \times 10^{-3}$ & $6.77 \times 10^{-2}$ \\
$40 \mathrm{wt} \%$ glycerol & 1099.3 & $3.72 \times 10^{-3}$ & $6.95 \times 10^{-2}$ \\
$20 \mathrm{wt} \%$ glycerol & 1046.9 & $1.76 \times 10^{-3}$ & $7.09 \times 10^{-2}$ \\
\hline
\end{tabular}

consequence the behaviour of the rising bubble pair. To minimise the effect of preceding bubbles, a flow rate of 1 $\mathrm{ml} / \mathrm{min}$ was chosen, based on earlier tests (Kong et al. 2019).

The recording was set at a frame rate $\left(f_{\text {rec }}\right)$ of $2000 \mathrm{~Hz}$, focused at a domain of $20 \times 50 \mathrm{~mm}$, yielding a resolution of $30 \mu \mathrm{m} /$ pixel. The recorded images were processed using an in-house Matlab program. Details can be found in Kong et al. (2019). The uncertainty in determining the bubble centre from the image processing is lower than $1 \%$ due to the high resolution. It should be noted that all cases were repeated for multiple times. However, the most difficult part is to make two bubbles detach perfectly at the same time. Milliseconds difference of detachment between the two bubbles of a pair would lead to a largely different interaction. In this study, picking from around 20 results, we normally have 5 sets of results, for which the time difference is within $1 \mathrm{~ms}$. The best one (minimum time difference cases, usually is $0.5 \mathrm{~ms}$ ) is then selected. Since the dynamic picture relies on one sensitive factor (detachment timing of the bubble pair), processing the best case instead of averaging a large set of data is chosen as the strategy of analysis. This strategy has also been widely used in studies of bubble dynamics.

\subsection{Data-processing procedures}

The velocity and acceleration of the rising bubble correspond, respectively, to the first and second derivative of the position obtained from image processing. From our experiments, we obtain the bubble position as a function of time and to obtain the velocity and acceleration of the bubble in principle numerical differentiation is required. As used in most of the experimental studies, the velocity and acceleration could in principle be obtained from

$$
\begin{aligned}
\frac{\mathrm{d} \tilde{y}\left(t_{i}\right)}{\mathrm{d} t} & =\frac{\tilde{y}_{i+1}-\tilde{y}_{i-1}}{2 \Delta t}+\mathcal{O}\left(\Delta t^{2}\right) \\
& =\frac{\mathrm{d} y\left(t_{i}\right)}{\mathrm{d} t}+\mathcal{O}\left(\frac{\epsilon}{\Delta t}\right)+\mathcal{O}\left(\Delta t^{2}\right),
\end{aligned}
$$

in which $\tilde{y}_{i}=y\left(t_{i}\right)+\epsilon . \tilde{y}_{i}, y_{i}(t)$ and $\epsilon$ are the measured quantity, the true quantity, and the measurement error, respectively. For small $\Delta t$, error amplification prevails (as shown in Fig. 2) that will be detrimental for the evaluation of the

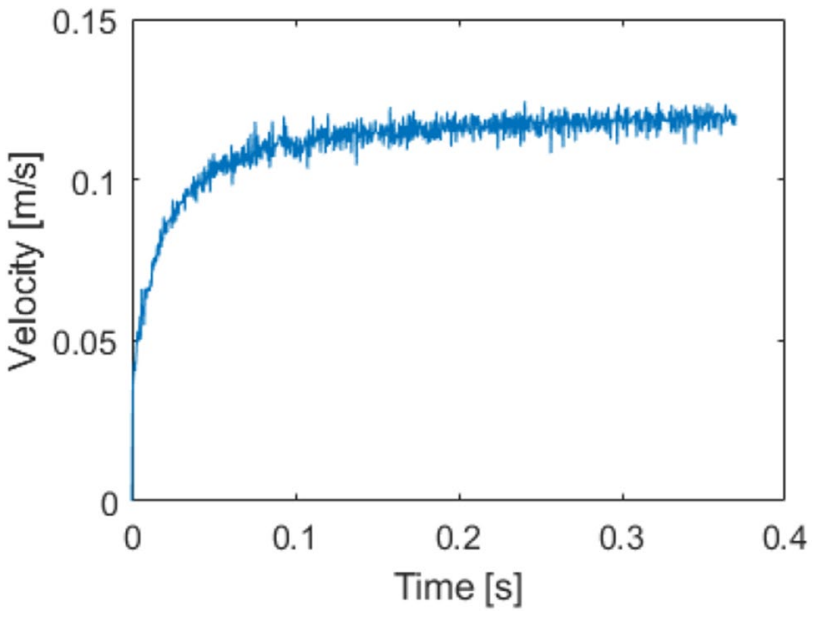

Fig. 2 Velocity of a rising bubble featuring strong velocity fluctuations. Data were obtained from the experiment of a $3.15 \mathrm{~mm}$ air bubble rising in $80 \%$ glycerol solutions

higher derivatives. To avoid the problem, a fitting method can be applied, which basically introduces smoothing.

There exists several fitting methods such as the leastsquares approach or fitting using cubic splines (De Vries et al. 2002b). Furthermore, smoothing spline fitting (Reinsch 1967) was introduced as a powerful tool to minimise the measurement noise when the derivatives of measured quantities are of interest. The basic idea is to find a fitting function balancing the desire of resembling the original data and that of resembling the underlying model.

Unfortunately, the fitting function is very sensitive to the choice of the smoothing parameter. Moreover, it is very challenging to choose the approximate value of the smoothing parameter (Aydın et al. 2013). To establish the aforementioned balance in a rational fashion, the method to determine the smoothing parameter was based on studies of De Boor et al. (1978), Hutchinson (1986) and Wahba (1983).

For a set of measured data $\left(x_{i}, y_{i}\right)$, the underlying relation of the data $\mathrm{g}\left(x_{i}\right)$ and measurement noise $\epsilon_{i}$ is related as

$y_{i}=g\left(x_{i}\right)+\epsilon_{i}$,

defining the so-called "de Boor formulation". To recover the relation $g\left(x_{i}\right)$, a function $f$ can be constructed. $g=f_{\mathrm{p}}$, where p equals to the value obtained by minimising the following function:

$p \sum_{i=1}^{N}\left(\frac{y_{i}-f\left(x_{i}\right)}{\delta y_{i}}\right)^{2}+(1-p) \int_{x_{1}}^{x_{N}}\left(f^{(m)}(t)\right)^{2} \mathrm{~d} t$

The first term is the normalised residual. The second term is the roughness penalty. In extreme cases of $p=0$, the fit is the least-squares approximation, which is the smoothest 
fitting, whereas for $p=1$, the fit is the spline interpolant, which links all sampling data. The constructed function $f_{\mathrm{p}}$ is considered as the underlying relation function $g(x)$. Based on this derivation, several approaches have been developed such as the corrected Akaike information criterion (AICC) (Hurvich et al. 1998), the Vapnik's measure (statistical learning theory) (VM) (Cherkassky and Mulier 2007), and the generalized cross validation (GCV) (Wahba 1983). In the present study, the AICC approach is adopted to automatically determine the smoothing parameter $p$ (Krakauer and Krakauer 2012). To calculate the velocity and acceleration, derivatives of the fitted centre data are calculated analytically:

$v=\frac{\mathrm{d} f}{\mathrm{~d} t}$

$\left.a=\frac{\mathrm{d}^{2} f}{\mathrm{~d} t^{2}},\right\}$

To validate the data-processing method in the present study, DNS results were used as a well-defined case (Mirsandi et al. 2018; Kong et al. 2019), see Fig. 3. The bubble velocity and interface position can be extracted from the simulation results readily. The snapshots of the simulated bubble were exported as experimental images. We use binary snapshots of a bubble, since the accuracy of detection of the bubble interface has been proven very high. Therefore, only the data processing is assessed, because other uncertainty sources are expected to be of minor influence.

The bubble velocity determined from the synthetic images is compared to the velocity obtained from the DNS in Fig. 4. The residual shows that the digital image analysis (DIA) and the data processing are reliable and the smoothing

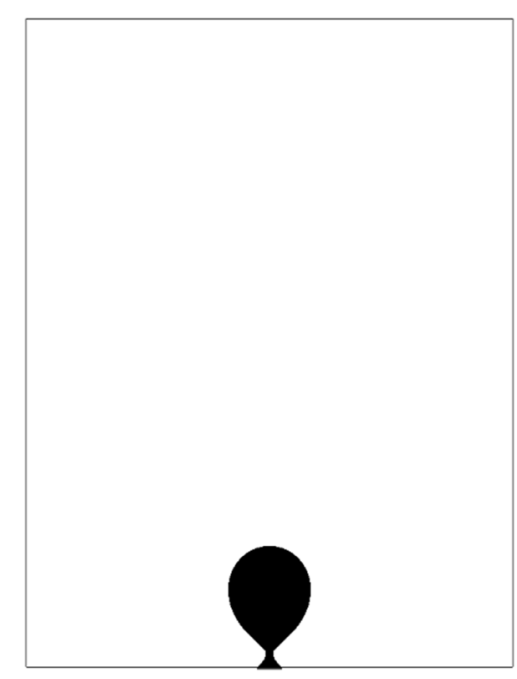

Fig. 3 Snapshot of bubble obtained from DNS simulation. The simulation was conducted with the properties of a $3.18 \mathrm{~mm}$ air bubble rising in $60 \%$ glycerol solutions

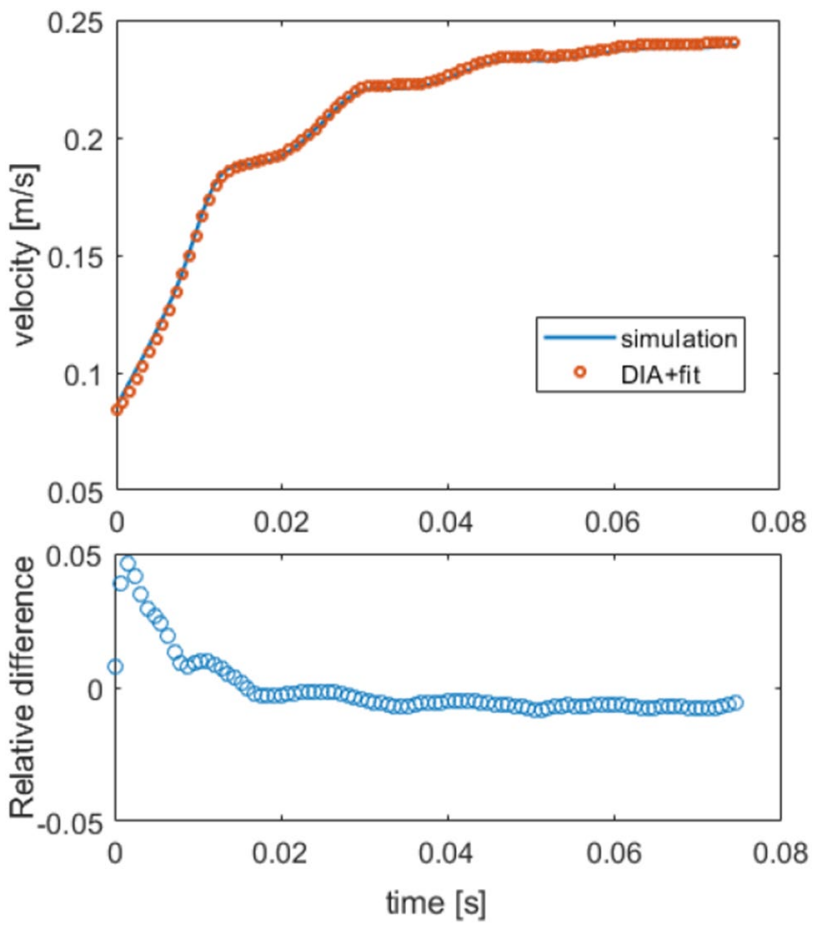

Fig. 4 Validation of the data-processing procedure. Velocity obtained from the adapted processing procedure and velocity extracted from DNS simulation are plotted together in the upper figure; relative difference is plotted in the lower figure

method is capable of minimising the noise. The errors are in the order of $1 \%$, except for the initial moment up to 10 $\mathrm{ms}$, which is related to the detachment phase of the bubble. This has been demonstrated in a previous study (Kong et al. 2019).

\section{Results and discussion}

Studies on bouncing and coalescence have revealed that wakes play an important role (Duineveld 1998; Sanada et al. 2005). According to the study of Magnaudet and Mougin (2007), rising bubbles can be categorised into three groups (Fig. 5). Glycerol solutions with pre-selected viscosities are employed to obtain the desired bubble rise regime. Bubbles of small size $(R e)$ and (nearly) spherical shape (regimes a and $b$ ) have no vortex in the wake and rise in a rectilinear path; bubbles of larger size $(R e)$ and moderate deformation (regime c) have an axisymmetric standing vortex wake and rise following a rectilinear path; bubbles of even larger size $(R e)$ and pronounced deformation possess unstable wakes and rise following a complex three-dimensional path.

How a bubble pair behaves is a fundamental question for understanding the large-scale behaviour of bubble swarms. In the present study, the rising dynamics of pairs of spherical bubbles and spheroidal bubbles are 


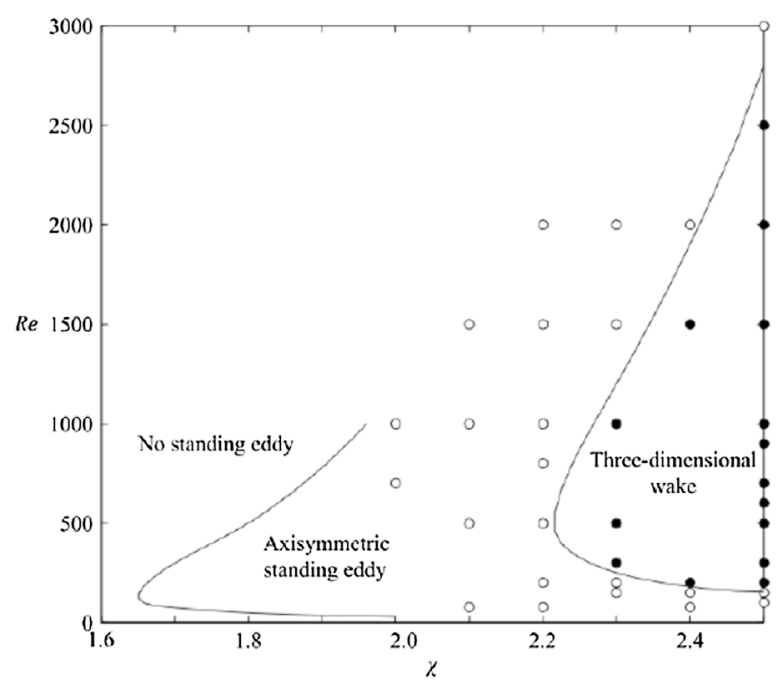

(a)

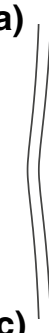

(c)

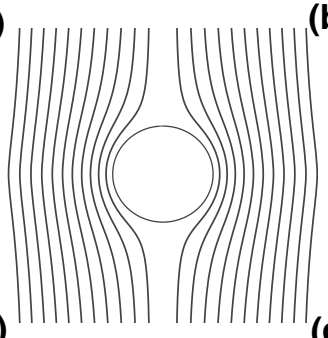

(b)

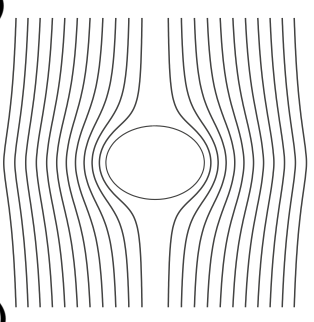

(d)

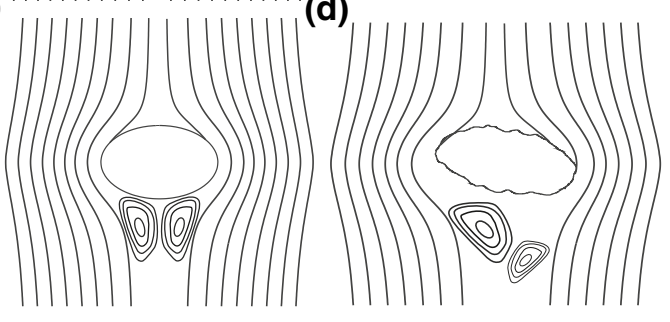

Fig. 5 Left: phase diagram showing the wake structure of rising bubbles against $(\operatorname{Re}, \chi)$ (defined the same as in Table 2) (Magnaudet and Mougin 2007); right: schematic representation of the wake of bubbles. a-d Correspond to bubbles in Table 2

experimentally studied to investigate the interaction. The dimensionless distance of two centres of mass is defined as $S=2 s / D_{\text {eq }}$. It should, however, be noted that the approach or separation of the pair of bubbles does not always reveal the interaction of the bubbles, because for large bubbles, the unstable path can result in the relative movement of a pair of bubbles, which obviously is not due to the existence of the second bubble. It should be noted that the bubble sizes are carefully checked to make sure that the size difference between the two bubbles of the pair as well as the size of the corresponding single bubble is approximately $\pm 0.03 \mathrm{~mm}$.

The different bubble types and corresponding dimensionless numbers are listed in Table 2. Based on their $M o, E o$, and Re numbers, the classification provided by the Grace diagram (Clift et al. 2005) gives that types a, $\mathrm{b}$, and $\mathrm{c}$ as well as d are in the spherical, ellipsoidal, and wobbling regime, respectively. The following sections are organised according to the results obtained per bubble type.

\subsection{Spherical bubbles (type a)}

The interactions of spherical bubbles are represented in Fig. 6a. It is clear that irrespective of the initial separation distance, all the bubbles repel each other after an initial short period of attraction. The repulsion is more prominent for a smaller initial distance.

Velocities and deformation are calculated by implementing the image and data-processing procedure, as discussed in Sect. 2.2. Figure 7 displays the rise velocity, deformation, and separation distance as a function of the height for different initial separation distances. The mirrored horizontal velocity and overlapped deformation reveal the symmetry of the experiments. As shown in Fig. 6a, the distance between the bubbles comprising the pair all increase with the rising height. It can be seen that a smaller initial distance leads to a more pronounced separation and larger horizontal velocity. The curves for deformation of the bubbles are identical and equal to that of a single bubble, which reveals that the interaction has no impact on the shape for such relatively rigid bubbles. Furthermore, the vertical velocities of both bubbles
Table 2 Characteristic values and dimensionless numbers of bubbles

\begin{tabular}{llrlllllll}
\hline & $D_{\mathrm{eq}}$ & \multicolumn{1}{c}{$R e$} & $\log (\mathrm{Mo})$ & Eo & We & Oh & $\chi$ & $C a$ & Type \\
\hline $80 \mathrm{wt} \%$ glyc. & 3.15 & 7.5 & -3.4 & 1.81 & 0.8 & 0.17 & 1.08 & 0.1 & $\mathrm{a}$ \\
$60 \mathrm{wt} \%$ glyc. & 3.18 & 84.9 & -6.4 & 1.69 & 3.4 & 0.03 & 1.5 & 0.04 & $\mathrm{~b}$ \\
$40 \mathrm{wt} \%$ glyc. & 3.23 & 286.3 & -8.3 & 1.62 & 5.0 & 0.01 & 2.1 & 0.016 & $\mathrm{c}$ \\
$20 \mathrm{wt} \%$ glyc. & 3.22 & 574.6 & -9.6 & 1.50 & 4.3 & 0.005 & 2.5 & 0.007 & $\mathrm{~d}$ \\
\hline
\end{tabular}

The rising behaviours correspond to Fig. 5. Dimensionless numbers are defined as: $E o=\rho_{l} g D_{\text {eq }}^{2} / \sigma$, $W e=\rho_{l} U^{2} D / \sigma, R e=\rho_{l} U D / \mu, O h=\mu /\left(\rho_{l} \sigma D_{\text {eq }} / 2\right)^{1 / 2}, M o=g \mu_{l}^{4} /\left(\rho \sigma^{3}\right), C a=\mu U / \sigma, \chi=R_{1} / R_{2}\left(R_{1}\right.$ and $R_{2}$ are the semi-major and semi-minor axes, respectively) 
Fig. 6 Trajectories of bubble pair rising in viscous liquids and the corresponding trajectories of single isolated rising bubbles. a-d Correspond to bubbles in Table 2 and in Fig. 5. a Bubbles in $80 \%$ glycerol solutions with an initial distance of $4 \mathrm{~mm}, 6$ $\mathrm{mm}$, and $7 \mathrm{~mm}$, respectively. Time interval of images superposition is $25 \mathrm{~ms}$. b Bubbles in $60 \%$ glycerol solutions with an initial distance of $4 \mathrm{~mm}, 6 \mathrm{~mm}$, and $7 \mathrm{~mm}$, respectively. Time interval of images superposition is $10 \mathrm{~ms}$. c Bubbles in $40 \%$ glycerol solutions with an initial distance of $4 \mathrm{~mm}, 6 \mathrm{~mm}$, and 7 $\mathrm{mm}$, respectively. Time interval of images superposition is 10 ms. d Bubbles in 20\% glycerol solutions with an initial distance of $4 \mathrm{~mm}$. Time interval of images superposition is $10 \mathrm{~ms}$

Fig. 7 Rising behaviour of a pair of bubbles in $80 \%$ Glycerol liquids. From top to bottom, the initial distance amounts 4 $\mathrm{mm}, 6 \mathrm{~mm}$, and $7 \mathrm{~mm}$; in each column, the vertical velocity, horizontal velocity, deformation, and separation distance against the rising height are shown. Blue line: the left rising bubble; orange line: the right rising bubble; black line: the single isolated rising bubble (a)
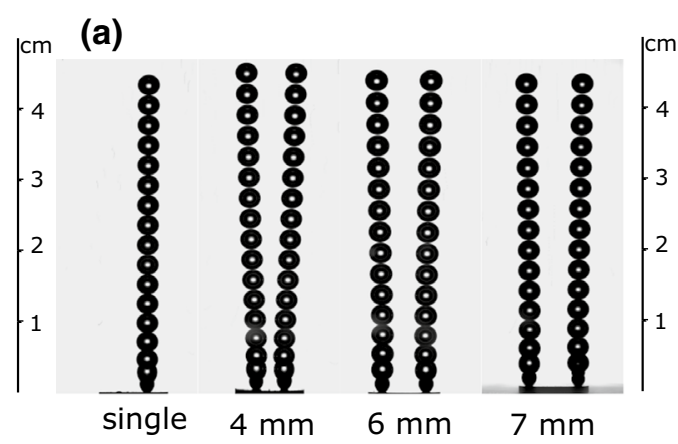

(b)

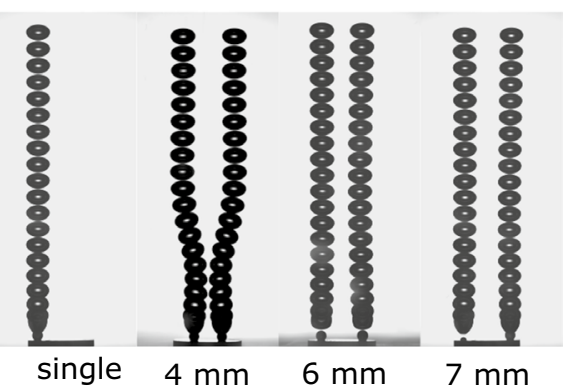

(c)

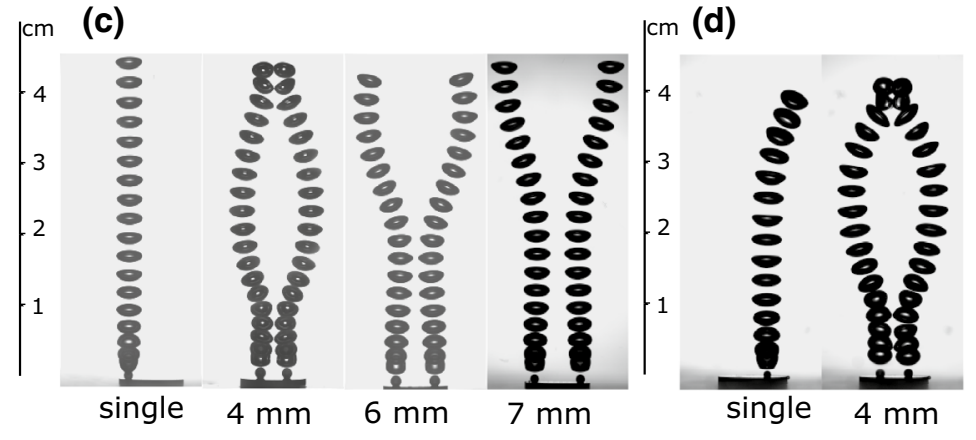

vertical velocity horizotal velocity

deformation separation distance
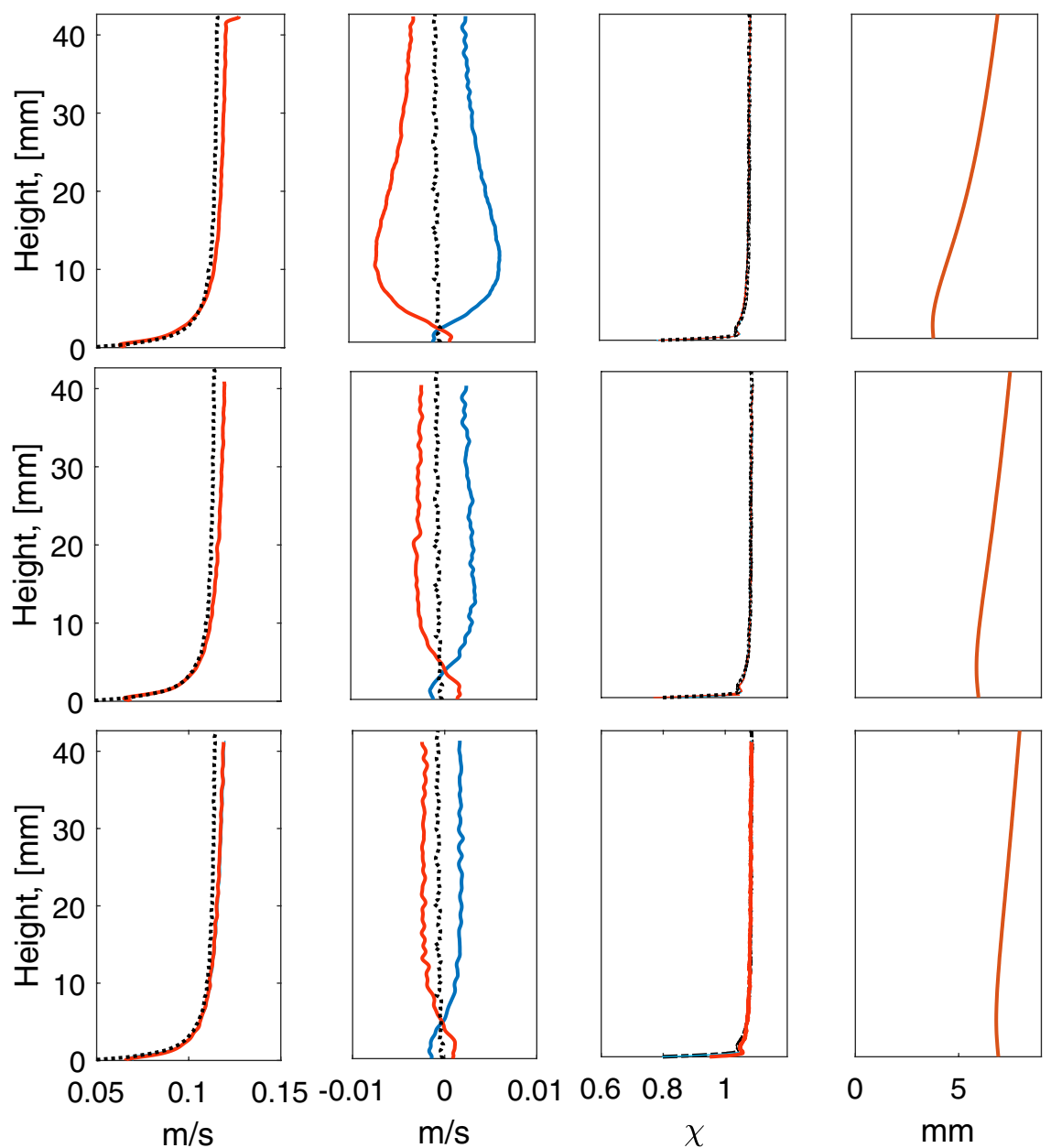
are identical. However, surprisingly, the bubble pairs rise faster in comparison with a single rising bubble.

Previous studies, both experimental (Kok 1993) and theoretical (Van Wijngaarden and Jeffrey 1976), have reported attraction of spherical bubbles. Due to the high strain rate in the liquid between the bubbles, the rise velocity is predicted to be lower than a single rising bubble. Later, Legendre et al. (2003) conducted a DNS for a pair of fixed spherical bubbles. Their results show that spherical bubbles tend to attract if the Reynolds number is large, whereas such bubbles repel if the Reynolds number is small. In case of the study of Kok (1993), the Reynolds number was 240 , Weber number was 0.76 . In the present study, the Reynolds number is 7.5 , and the Weber number is 0.8. Moreover, Legendre et al. (2003) indicated that the repulsive interaction and the higher rise velocity are associated with the distribution of vorticity, which is altered by the interaction.

\subsection{Deformed bubbles (type b)}

Type $b$, deformed bubbles are no longer spherical, but have a relatively stable ellipsoidal shape. The rising behaviour of these bubbles is presented in Fig. 6b. For initial distances of $6 \mathrm{~mm}$ and $7 \mathrm{~mm}$, the interaction is very weak and both bubbles rise almost identically to a single rising bubble, which is especially apparent from the vertical rise velocity and the deformation. However, the interaction is prominent for the case of an initial distance of $4 \mathrm{~mm}$. The bubbles initially approach before separating and during the interaction, especially during separation these bubbles seem to rotate slightly. Strikingly, all bubbles seem to attain the same final properties.

Velocities, deformation, and the separation distance are shown in Fig. 8. It is clear that the separation distance only changes slightly for initial distances of $6 \mathrm{~mm}$ and $7 \mathrm{~mm}$, which is different in comparison with spherical bubbles. The deformation curves are also identical for those two cases, whereas for the initial distance of $4 \mathrm{~mm}$, the terminal rise velocity and final shape are delayed, before reaching the same values as for a single rising bubble. Similar to the spherical bubble, the horizontal velocities reveal the extent of interaction. It is obvious that the horizontal velocities are larger for an initial distance of $4 \mathrm{~mm}$. For the $7 \mathrm{~mm}$ case, no horizontal velocity is obtained, indicating the absence
Fig. 8 Rising behaviour of a pair of bubbles in $60 \%$ Glycerol liquids. From top to bottom, the initial distance amounts 4 $\mathrm{mm}, 6 \mathrm{~mm}$, and $7 \mathrm{~mm}$. In each column, the vertical velocity, horizontal velocity, deformation, and separation distance against the rising height is shown. Blue line: the left rising bubble; orange line: the right rising bubble; black dashed line the single isolated rising bubble vertical velocity
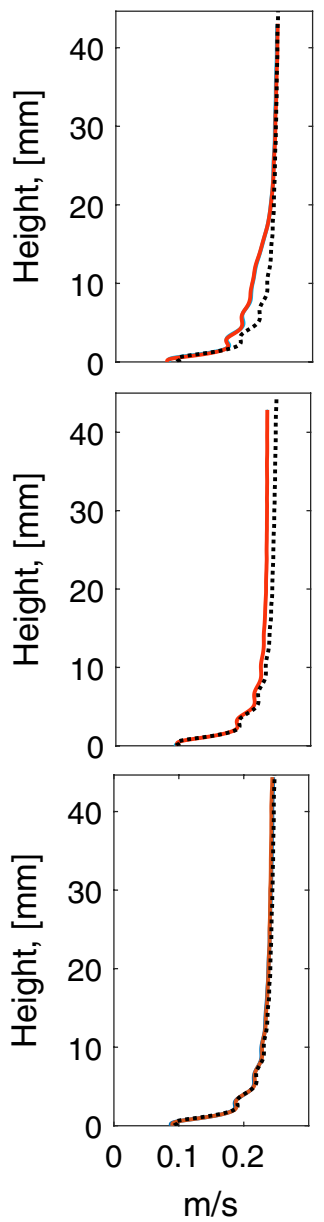

horizotal velocity
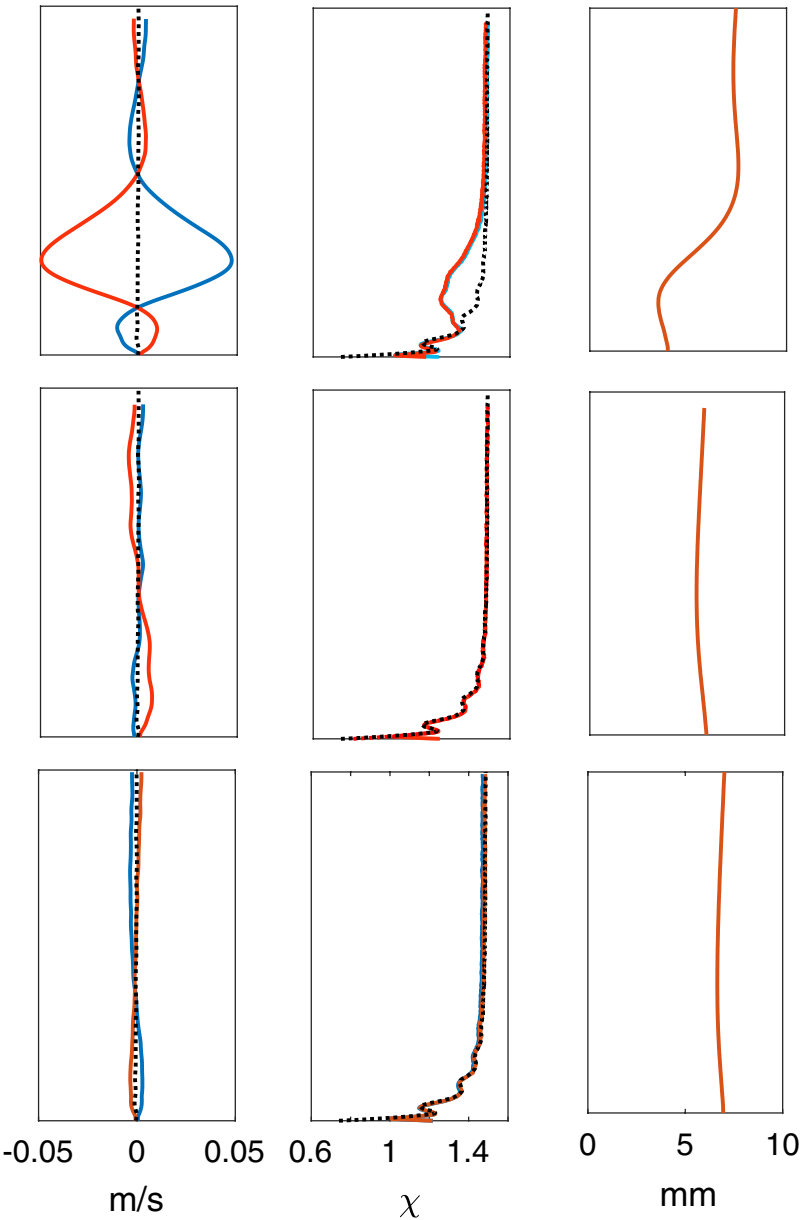
of interaction and consequently all other parameters are the same as for a single rising bubble. For the $6 \mathrm{~mm}$ case, a part of the vertical rise velocity seems to be transferred to the horizontal direction while not affecting the shape. This can be mainly attributed to the smaller distance. On the other hand, the vertical velocities between the bubbles composing the pair and the single isolated bubble are identical during the earlier and later stages, but are lower in the other stage.

\subsection{Deformable bubbles (types c, d)}

Compared with deformed bubbles and spherical bubbles, deformable bubbles are less rigid. The bubbles will experience shape oscillations (Kong et al. 2019) due to the wake behaviour. As shown in Fig. 6c, d, multiple bubble encounters occur for deformable bubble pairs and the deformation itself is strongly altered by the interaction.

Based on the discussion of Sects.3.1 and 3.2, we can conclude that the interaction is negligible if both the vertical velocity and deformation are identical to the corresponding single bubble, and arguably if only the deformation is identical. In Fig. 9, for all the cases of initial separation distance, the bubbles first approach each other before separating. The velocity and deformation are identical to that of a single rising bubble, up to the point, where the bubbles meet and separate again. After this encounter and during the subsequent separation, the shape oscillations and rise velocity start to deviate from the single bubble rise. This onset of bubble interaction occurs earlier for smaller initial distance. Both the deformation and the rise velocity are suppressed with the interaction of the two bubbles.

As a complementary case, a bubble pair rising in $20 \%$ glycerol (see Fig. 6d) shows the interaction of a bubble pair with an unstable wake. Instead of the development of a 3D trajectory for a single bubble rise, the bubble pair has an extended period of an in plane movement.

From Figs. 8, 9, and 10, it can be seen that the deformation of bubble pairs is weaker in comparison with the single bubble rising (note that the larger deformation of the single rising bubble in Fig. 10 is attributed to the underestimation of deformation due to projection of a three-dimensional
Fig. 9 Rising behaviour of a pair of bubbles in $40 \%$ Glycerol liquids. From top to bottom, the initial distance amounts 4 $\mathrm{mm}, 6 \mathrm{~mm}$, and $7 \mathrm{~mm}$. In each column, the vertical velocity, horizontal velocity, deformation, and separation distance against the rising height is shown. Blue line: the left rising bubble; orange line: the right rising bubble; black dashed line: the single isolated rising bubble

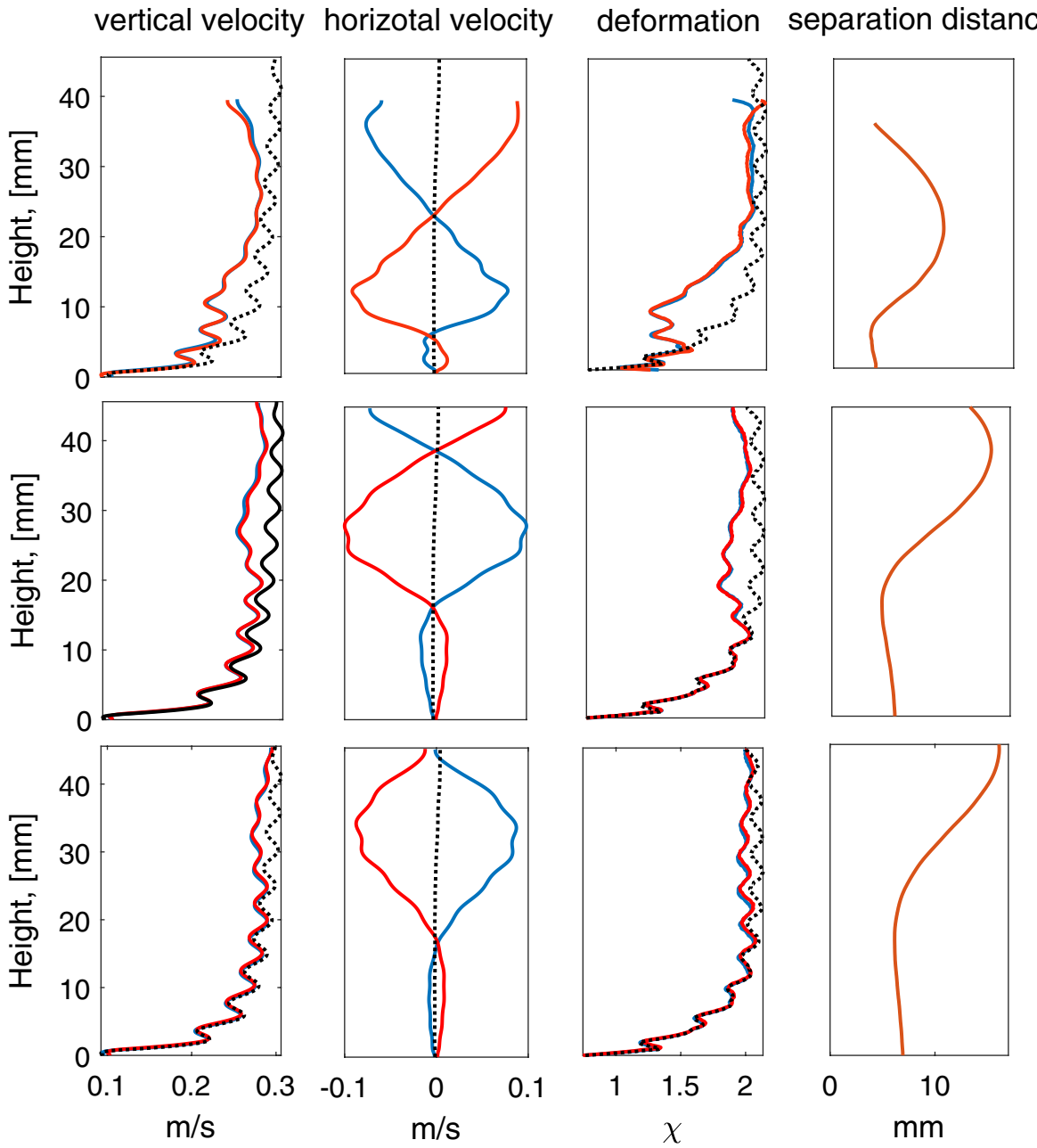


Fig. 10 Rising behaviour of a pair of bubbles in $20 \%$ glycerol liquid. Curves are given for an initial distance $4 \mathrm{~mm}$; in each column the vertical velocity, horizontal velocity, deformation, and separation distance against the rising height is given. Blue line: the left rising bubble; orange line: the right rising bubble; black dashed line: the single isolated rising bubble
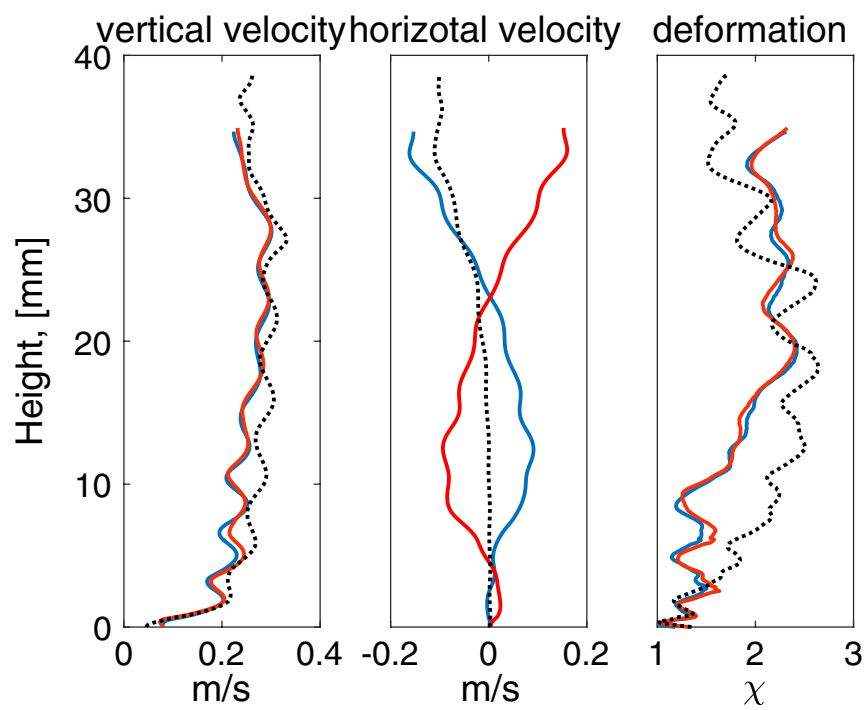

separation distance

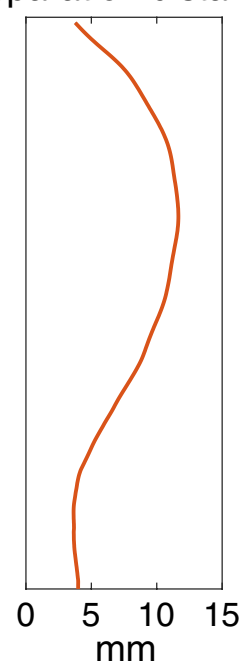

object). The interaction of the bubble pair seems to suppress the extent of deformation.

\subsection{Drag and lift coefficients}

The forces exerted on the bubble due to the presence of the second bubble provide the key information to understand the interaction. So far the only study that calculated the forces based on measurements are the studies of Shew et al. (2006) and Shew and Pinton (2006) owing to the high accuracy of their velocity measurements. In view of the high accuracy of velocity our measurements (in Sect. 2.2), we expect to obtain reliable force evaluations. The method used in studies of Shew et al. (2006) is based on the generalized Kirchhoff equations (Mougin and Magnaudet 2002; Howe 1995) given by

$$
\left.\begin{array}{r}
\rho V\left(\mathbf{A} \cdot \frac{\mathrm{d} \mathbf{U}}{\mathrm{d} t}+\boldsymbol{\Omega} \times \mathbf{A} \cdot \mathbf{U}\right)=\mathbf{F}_{\mathbf{e}}, \\
\rho V\left(\mathbf{D} \cdot \frac{\mathrm{d} \boldsymbol{\Omega}}{\mathrm{d} t}+\boldsymbol{\Omega} \times(\mathbf{D} \cdot \boldsymbol{\Omega})+\mathbf{U} \times(\mathbf{A} \cdot \mathbf{U})\right)=\boldsymbol{\Gamma}_{\boldsymbol{e}},
\end{array}\right\}
$$

where $\mathbf{F}_{\mathrm{e}}$ and $\boldsymbol{\Gamma}_{\boldsymbol{e}}$ are the external force and torque on a body, respectively. $\mathbf{A}$ and $\mathbf{D}$ stand for the translational and rotational added mass coefficients, respectively. $\boldsymbol{\Omega}$ is the angular velocity and $\mathbf{U}$ is the translational velocity. This equation expresses the balance of momentum and angular momentum on a body translating and rotating in a fluid. It is, therefore, possible to estimate the force and torque acting on the bubble if the trajectories and orientation of the bubble are available by implementing this equation (Fig. 11).

If the shape of the bubble is axis-symmetric together with the accepted assumption that the minor axis of the bubble is nearly aligned with its velocity (Mougin and Magnaudet 2001; De Vries et al. 2002a; Ellingsen and

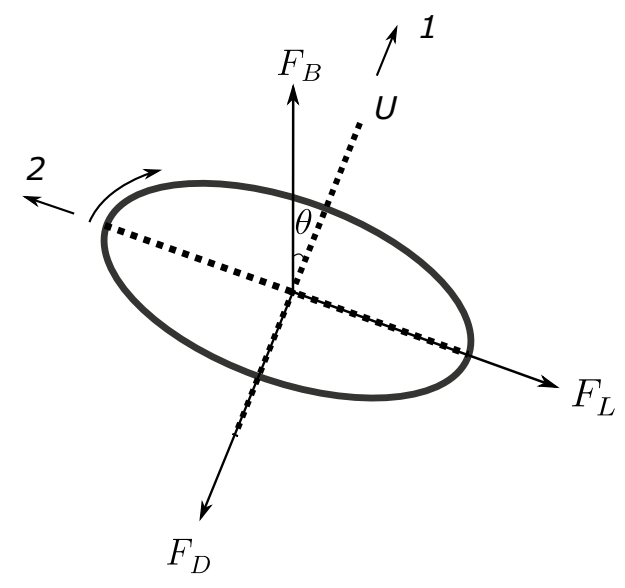

Fig. 11 Schematic representation of forces exerted on the bubble. $F_{\mathrm{B}}, F_{\mathrm{L}}$, and $F_{\mathrm{D}}$ are the buoyancy force, lift force, and drag force, respectively. $\theta$ is the pitch angle. $U$ is the bubble velocity. 1 and 2 are attached on the moving frame of the bubble

Risso 2001) as well as a moving frame chosen to be fixed on the axes of the bubble, Eq. 5 can be shown to reduce to

$\left.\begin{array}{l}\rho V A_{11} \frac{\mathrm{d} U}{\mathrm{~d} t}=F_{\mathrm{D}}+F_{\mathrm{B} 1}, \\ \rho V \Omega A_{11} U=F_{\mathrm{L}}+F_{\mathrm{B} 2},\end{array}\right\}$

where $\Omega=-\frac{\mathrm{d} \theta}{\mathrm{d} t}, F_{\mathrm{B} 1}=\rho V g \cos \theta, F_{\mathrm{B} 2}=\rho V g \sin \theta$, and $\theta=\operatorname{atan}\left(U_{x} / U_{z}\right) . A_{11}$ is the added mass tensor. $A_{11}$ is a function of the bubble shape/geometry, given by Lamb (1993):

$\left.\begin{array}{r}A_{11}=\alpha /(2-\alpha), \\ \alpha=2 /\left(1-\chi^{-2}\right)\left(1-\chi^{-2} / \sqrt{1-\chi^{-2}} \arcsin \left(\sqrt{1-\chi^{-2}}\right)\right) .\end{array}\right\}$ 
By implementing the force calculation, the drag force coefficient and lift force coefficient are calculated based on

$\left.\begin{array}{l}C_{1}=F_{\mathrm{L}} /\left(\pi R_{2}^{2} \rho U^{2} / 2\right), \\ C_{\mathrm{D}}=F_{\mathrm{D}} /\left(\pi R_{1}^{2} \rho U^{2} / 2\right),\end{array}\right\}$

where $R_{1}, R_{2}$ are the semi-major axis and semi-minor axis, respectively.

The drag and lift coefficients are shown in Fig. 12 for spherical bubbles, deformed bubbles, and deformable bubbles. It is stressed here that the results obtained for deformable bubbles should be regarded as qualitative, as we have discussed in Sect. 3.3. In addition, the acceleration phase will be excluded from the analysis, as the obtained data are prone to error amplification, as discussed in Sect. 2.2.

Spherical bubbles (type a) rising side-by-side have been investigated in a few studies (Kok 1993; Van Wijngaarden and Jeffrey 1976; Legendre et al. 2003). The lift force coefficient assuming potential flow (Van Wijngaarden and Jeffrey 1976) is given by
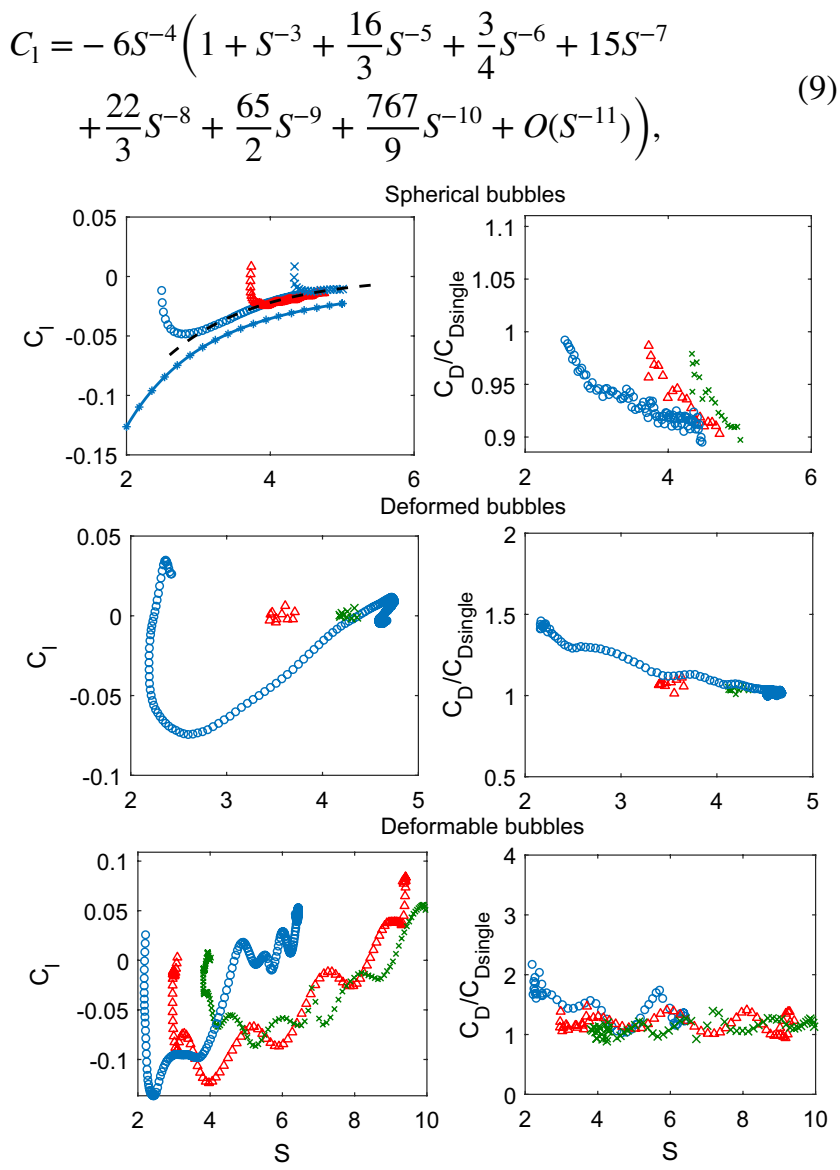

Fig. 12 Lift coefficients and drag coefficients of different types of bubbles vs. separation distances. Blue circle: data from $4 \mathrm{~mm}$ case. Red triangle: data from $6 \mathrm{~mm}$. Green cross mark: data from $7 \mathrm{~mm}$ case. In the first row and first column, dashed line: a fitting curve; dashed asterisk line: the Stokesian theoretical prediction (Eq. 10) (Legendre et al. 2003) corresponding to attraction type of interaction. As discussed by Legendre et al. (2003), this result is only valid at large Reynolds number. An analytical expression (Legendre et al. 2003) incorporating shear-free boundary with an analytical theory for sedimenting low-Reynolds-number particles (Vasseur and Cox 1977) is given by

$C_{l}(\operatorname{Re}, \operatorname{ReS})=\frac{32}{\operatorname{Re}^{2} S^{2}}\left[1-\left(1+\frac{\operatorname{ReS}}{4}\right) e^{-\operatorname{ReS} / 4}\right]$,

the expression is included in Fig. 12. The curves obtained from our experiments for different initial distances follow the same trend. Moreover, the agreement between our data and the analytical results is satisfactory. The discrepancy is due to the bubble Reynolds number in the present study amounts to 7.5, which slightly exceeds the value of Legendre et al. (2003).

In this case, the magnitude of the lift coefficient declines exponentially with a long tail. This reveals that the repulsive interaction decays until a certain separation distance has been reached. In addition, the study of Legendre et al. (2003) reported a drag coefficient ratio of $90 \%$ for interacting bubbles, which is in good agreement with our results.

For the deformed bubbles (type b) rising side-by-side, in comparison with the spherical bubbles (type a), the Weber number is higher than 1, which gives rise to shape deformation. The deformation and the existence of torques exerted on the bubbles complicate the interaction. As shown in Sect. 3.2, the motion of rising bubbles consists of translational and rotational motions.

We will subsequently analyse the effect of bubble interaction on the energy changes, which requires estimations of the kinetic energy, rotational energy, and surface energy. Limited by our experimental method, the energy dissipation is not considered. However, this can be further analysed and justified by DNS in future work. The gravitational potential energy is neglected as well. The kinetic energy of lateral motion $\left(E_{\mathrm{kx}}\right)$ reads (Jeong and Park 2015; Newman 1977)

$E_{\mathrm{kx}}=\frac{2}{3} \rho R_{2} R_{1}^{2} U_{x}^{2} \frac{\beta}{2-\beta}$,

where $\rho$ is the density of the surrounding liquid. $R_{1}$ and $R_{2}$ are the semi-major and minor axes of bubbles, respectively. $U_{x}$ is the lateral velocity. $\beta=\frac{1}{e^{3}}\left[\sqrt{1-e^{2}} \sin ^{-1} e-e\left(1-e^{2}\right)\right]$, $e=\sqrt{1-\chi^{-2}}$ (Newman 1977).

Likewise, the rotational energy $\left(E_{\text {rot }}\right)$ can be estimated from

$E_{\mathrm{rot}}=\frac{1}{2} k I \Omega^{2}$,

i n $\quad$ wh ich $I=\frac{4}{15} \rho \pi R_{2} R_{1}^{2}\left(R_{1}^{2}+R_{2}^{2}\right) \quad$ and $\quad k=$ $\frac{\left(R_{1}^{2}-R_{2}^{2}\right)^{2}(\alpha-\beta)}{\left(R_{1}^{2}+R_{2}^{2}\right)\left[2\left(R_{1}^{2}-R_{2}^{2}\right)-\left(R_{1}^{2}+R_{2}^{2}\right)(\alpha-\beta)\right]}$. 
The surface energy $\left(E_{\text {sur }}\right)$ of the bubble depends on the surface tension $\sigma$ and the surface area $\mathrm{A}$ and can be estimated from

$E_{\text {sur }}=\sigma A$,

in which $A=\frac{\pi R_{1}^{2}}{2}\left(1-\frac{1-e^{2}}{e} \tanh ^{-1} e\right)$.

The total energy $\left(E_{\text {total }}\right)$ due to the bubble interaction is defined as

$E_{\text {total }}=\Delta E_{\mathrm{sur}}+E_{\mathrm{kx}}+E_{\mathrm{rot}}$.

In Fig. 13, the energy contributions are plotted as a function of dimensionless time.

Together with the altered deformation described by a ratio of aspect ratios $\left(\chi / \chi_{\text {single }}\right)$, the aligned tendency of the curves reveals that the deformation is related to the bubble interaction.

As shown in Fig. 13, most of the energy from the interaction feeds into a suppression of the surface energy, where additionally the lateral kinetic energy $E_{\mathrm{kx}}$ and rotational energy $E_{\text {rot }}$ are orders of magnitude smaller. In particular, the rotational energy $E_{\text {rot }}$ is negligible. Based on the energy analysis, we find out that even though it does not seem very significant in Fig. 6, the deformation instead of the repulsion dominates in the case of strong interaction. Risso (2018) pointed out that the bubble agitation is surprisingly independent of bubble interaction, even at significant gas volume fraction. This has been found in several studies (Martínez-Mercado et al. 2007; Riboux et al. 2010; Colombet et al. 2015). In the present study, the dominance of the deformation could be a factor that leads to that phenomenon.

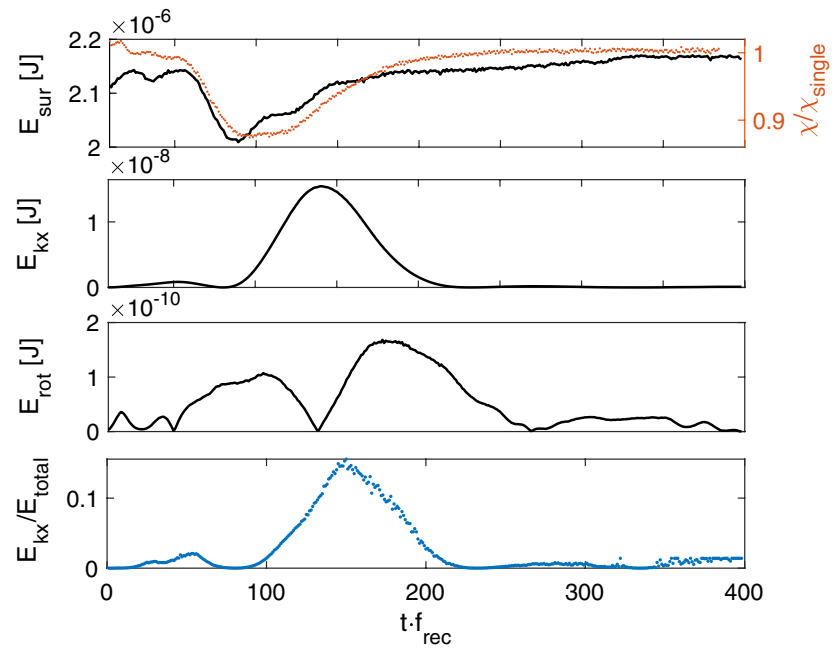

Fig. 13 Bubble energy contributions vs. dimensionless time [time is normalised by recording frequency $\left.\left(f_{\text {rec }}\right)\right]$ for an initial bubble distance of $4 \mathrm{~mm}$. The surface energy (single rising bubble included as a reference), lateral kinetic energy, rotational energy, and the ratio of the kinetic energy to the total energy are shown in the top-to-bottom sub-figures
Moreover, the time scale of relaxation of the altered deformation is similar to the decay of the surface oscillation in the order of $10^{-1} \mathrm{~s}$, which is correlated with the Ohnesorge number (Kong et al. 2019). This might indicate that the change of surface energy is due to viscous dissipation enforced by bubble interaction, after which it returns to the initial value when the stable separation distance is reached.

However, the lateral motion is significant as well, because it determines the separation distance which is a key parameter for the bubble swarm effect. For lift and drag force calculations, the deformation effect was neglected. Therefore, we provide an estimate of the deviation due to this neglect. From Fig. 8, it follows that the deformation is around 10\% of its terminal shape during the interaction. A 5\% deviation of the calculation of the lift and drag force is caused consequently by the neglect of the deformation. A similar estimate can be found in the study of Shew et al. (2006).

From Fig. 12, it can be seen that the results obtained for an initial distance of $4 \mathrm{~mm}$ reasonably cover the results for the other two cases (initial distances of $6 \mathrm{~mm}$ and $7 \mathrm{~mm}$ ). Specifically, the drag coefficient ratio obtained for initial distances of $6 \mathrm{~mm}$ and $7 \mathrm{~mm}$ are located on the curve of the $4 \mathrm{~mm}$ case. On the other hand, in terms of lift coefficient, 7 $\mathrm{mm}$ case is attached on the curve of $4 \mathrm{~mm}$, whereas $6 \mathrm{~mm}$ is outlying. As we have discussed in Sect. 3.2, even though the lift coefficient reveals that there is negligible interaction, the reduced rise velocity indicates the existence of interaction.

In fact, this is a sign of the torque effect. Apart from the force calculations that are simplified based on the assumption that the minor axis is aligned with the velocity, the second equation of (5) accounts for the torque effect, which reduces to Eq. (15):

$D_{33} \frac{\mathrm{d} \Omega}{\mathrm{d} t}+\left(A_{22}-A_{11}\right) V_{1} V_{2}=-\Gamma_{3}$.

The first term of the left side is the rotational acceleration. The second term on the left side accounts for the restoring torque, trying to realign the orientation of the bubble with the orientation of the velocity, as discussed in Magnaudet (2015), for a single bubble. Suppose a bubble is rising with a velocity, $\mathbf{U}=u_{1} \mathbf{e}_{1}+u_{2} \mathbf{e}_{2}$, the minor and major axes are along the $\mathbf{e}_{1}, \mathbf{e}_{2}$ directions, respectively. The corresponding components of the inertial tensor yield $A_{11}>A_{22}$. The inertial torque is $\Gamma \mathbf{e}_{\mathbf{3}}=-\mathbf{U} \times(\mathbf{A} \cdot \mathbf{U}), \Gamma=\left(A_{11}-A_{22}\right) U_{1} U_{2}>0$. This torque tends to align the velocity with its minor axis. This has earlier been neglected in calculations based on the alignment assumption.

Therefore, a further check addressing this particular assumption is carried out. The orientation of the bubble and its velocity is shown in Fig. 14. There is a period that the bubble velocity does not align with the minor axis of the bubble. As shown in Fig. 14, the "restoring torque" due to the misalignment and the torque due to the rotational 


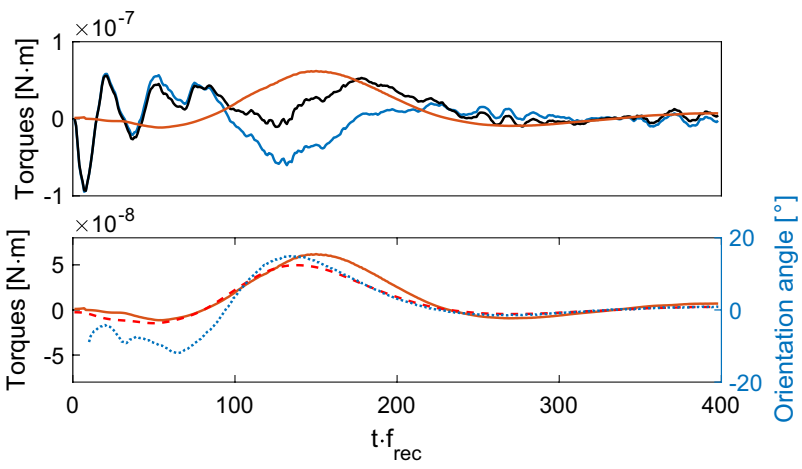

Fig. 14 Torques on the bubble and orientation of the rising bubble. Black line: sum of the left side of Eq. (15). Blue line: the first term of the left side of Eq. (15). Orange line: the second term of the left side of Eq. (15). Orange dashed line: The orientation of bubble. Blue dotted line: the orientation of the velocity

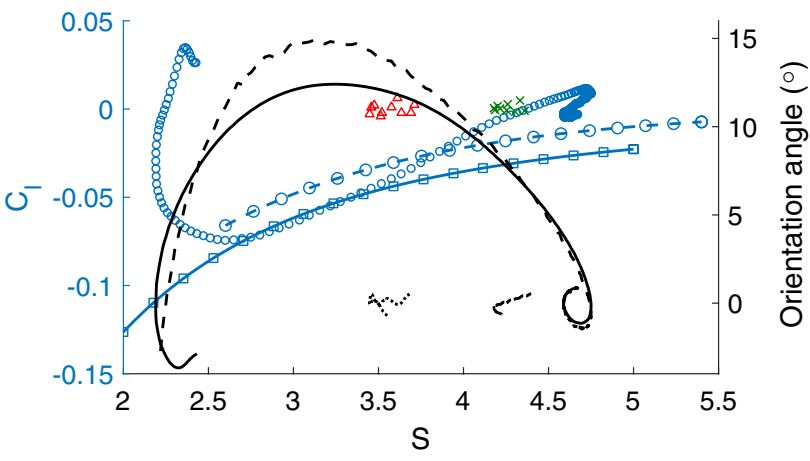

Fig. 15 Lift coefficients and drag coefficients of the bubble vs. the separation distance. Blue circle: $4 \mathrm{~mm}$ case. Red triangle: $6 \mathrm{~mm}$ case. Green cross mark: $7 \mathrm{~mm}$ case. $\square$ - stokesian theoretical prediction of lift coefficient for spherical bubbles (Eq. 10) (Legendre et al. 2003). - - - : lift coefficient for spherical bubbles obtained in the present study (type a). Black line: orientation of the velocity of the $4 \mathrm{~mm}$ case. Dashed line: orientation of the bubble of the $4 \mathrm{~mm}$ case. Dotted line: orientation of bubble of the $6 \mathrm{~mm}$ case. Dashed dotted line: orientation of the bubble of the $7 \mathrm{~mm}$ case

acceleration are of the same order. Moreover, the sum of the torque shows that a resulting torque appears when those two angles start recovering the alignment until the bubble rises broadside on. This torque hinders the rotation to the state of broadside on. This effect certainly allows that the bubble moves further in the lateral direction, in which case a larger separation distance is possible. Figure 15 reveals that the orientations of the bubble and the bubble velocity realign at the separation distance of 4.25 , which coincides with distance of $7 \mathrm{~mm}$. This suggests that at a distance of 7 $\mathrm{mm}$, the lateral motion is no longer driven by interactions and can dampen out naturally.

Comparing the lift coefficients of the $4 \mathrm{~mm}$ case and 6 $\mathrm{mm}$ case, the distinctions of bubble lift coefficients at the same separation distance reflect the complexity of interaction between deformed bubbles. The larger lift coefficient of $4 \mathrm{~mm}$ at the same separation distance as that of $6 \mathrm{~mm}$ case should be attributed to the torque effect. This also suggests that the equilibrium distance of side-by-side-deformed bubbles depend on the initial distance as well as the orientation.

In addition, as shown in Fig. 15, the magnitude of the lift coefficients surprisingly is of the same order of magnitude as for the spherical bubbles.

However, the lift coefficient of the deformed bubbles declines with a higher rate in comparison with the slow decay observed for the spherical bubbles. In this sense, deformed bubbles can establish a smaller separate distance between bubbles in comparison with spherical bubbles. Moreover, the ratio of the drag coefficient follows the opposite trend in comparison with spherical bubbles. The drag is higher for the rising bubble pair.

Finally, the results for the deformable bubbles (type c) will be discussed qualitatively. As indicated before, the results of deformable bubble pairs are less accurate. As shown in Fig. 12, the lift and drag coefficients are superimposed against the separation distance to some extent. The lift coefficients decline fast in a similar fashion as deformed bubbles (type b), even though with oscillations. Moreover, sign changes of the lift coefficient occur due to the path instability, which has been discussed in Sect. 3.3. On the other hand, the ratio of drag coefficients is generally above 1 , similar to deformed bubbles (type $b$ ), which reveal that the drag increases due to the bubble interaction.

\section{Conclusion}

In the present study, hydrodynamic interactions of side-byside rising bubble pairs are studied experimentally. Four different bubble types were studied by varying the liquid viscosity.

For spherical bubbles (type a), which have been studied prior to the present study, the behaviour of the bubble pair depends on the Re number. Bubbles at low Re number rise slightly faster than a single rising bubble and tend to repel each other. This behaviour is exactly opposite to rising pairs of spherical bubbles at high Re numbers, whose behaviour can be explained on the basis of potential flow theory. The repulsion of low Re number spherical bubble pairs exhibits exponential decay, leading to a larger equilibrium distance confirming earlier predictions by Legendre et al. (2003).

Interactions of deformed bubbles (type b) have been studied far less. The observations in this study show that deformed bubbles rise slower than a single rising bubble and overall bubble pairs repel each other. The lateral interaction of deformed bubbles is complex due to the torque acting on the deformed bubble, which depends on the initial distance 
and orientation. This lateral interaction declines faster in general, which reveals that a smaller equilibrium distance of the bubble pair is possible.

The results obtained for deformable bubbles are far more difficult to interpret. The results, however, do show the importance of shape deformation on the general pattern of bubble interaction. Interaction of the bubbles suppresses both the extent of deformation as well as the rise velocity. Our observations also reveal that the path instability can be triggered by the bubble interaction.

Acknowledgements We would like to thank the financial support from NWO TOP grant OND1356157 First-principles-based multi-scale modeling or transport in reactive three-phase flows. We would also like to thank the Industrial Partnership Programme i36 Dense Bubbly Flows that is carried out under an agreement between Akzo Nobel Chemicals International B.V., DSM Innovation Center B.V., SABIC Global Technologies B.V., Shell Global Solutions International B.V., Tata Steel Nederland Technology B.V.and Foundation for Fundamental Research on Matter (FOM), which is part of the Netherlands Organisation for Scientific Research (NWO).

Open Access This article is distributed under the terms of the Creative Commons Attribution 4.0 International License (http://creativecommons.org/licenses/by/4.0/), which permits unrestricted use, distribution, and reproduction in any medium, provided you give appropriate credit to the original author(s) and the source, provide a link to the Creative Commons license, and indicate if changes were made.

\section{References}

Aydın D, Memmedli M, Omay RE (2013) Smoothing parameter selection for nonparametric regression using smoothing spline. Eur $\mathbf{J}$ Pure Appl Math 6(2):222-238

Bouche E, Roig V, Risso F, Billet AM (2012) Homogeneous swarm of high-reynolds-number bubbles rising within a thin gap. Part 1. Bubble dynamics. J Fluid Mech 704:211-231

Bouche E, Roig V, Risso F, Billet AM (2014) Homogeneous swarm of high-reynolds-number bubbles rising within a thin gap. Part 2 . Liquid dynamics. J Fluid Mech 758:508-521

Bunner B, Tryggvason G (2003) Effect of bubble deformation on the properties of bubbly flows. J Fluid Mech 495:77-118

Cartellier A, Rivière N (2001) Bubble-induced agitation and microstructure in uniform bubbly flows at small to moderate particle reynolds numbers. Phys Fluids 13(8):2165-2181

Cherkassky V, Mulier FM (2007) Learning from data: concepts, theory, and methods. Wiley, New York

Clift R, Grace JR, Weber ME (2005) Bubbles, drops, and particles. Courier Corporation

Colombet D, Legendre D, Risso F, Cockx A, Guiraud P (2015) Dynamics and mass transfer of rising bubbles in a homogenous swarm at large gas volume fraction. J Fluid Mech 763:254-285

De Boor C, De Boor C, Mathématicien EU, De Boor C, De Boor C (1978) A practical guide to splines, vol 27. Springer, New York

De Vries A, Biesheuvel A, Van Wijngaarden L (2002a) Notes on the path and wake of a gas bubble rising in pure water. Int J Multiph Flow 28(11):1823-1835

De Vries J, Luther S, Lohse D (2002b) Induced bubble shape oscillations and their impact on the rise velocity. Eur Phys J B Condens Matter Complex Syst 29(3):503-509
Duineveld P (1995) Bouncing and coalescence of two bubbles in pure water. In: IUTAM symposium on waves in liquid/gas and liquid/ vapour two-phase systems. Springer, New York, pp 151-160

Duineveld P (1998) Bouncing and coalescence of bubble pairs rising at high Reynolds number in pure water or aqueous surfactant solutions. In: Fascination of fluid dynamics. Springer, New York, pp 409-439

Ellingsen K, Risso F (2001) On the rise of an ellipsoidal bubble in water: oscillatory paths and liquid-induced velocity. J Fluid Mech 440:235-268

Ern P, Risso F, Fabre D, Magnaudet J (2012) Wake-induced oscillatory paths of bodies freely rising or falling in fluids. Ann Rev Fluid Mech 44:97-121

Esmaeeli A, Tryggvason G (2005) A direct numerical simulation study of the buoyant rise of bubbles at o (100) Reynolds number. Phys Fluids 17(9):093303

Gumulya M, Utikar R, Evans G, Joshi J, Pareek V (2017) Interaction of bubbles rising inline in quiescent liquid. Chem Eng Sci 166:1-10

Hallez Y, Legendre D (2011) Interaction between two spherical bubbles rising in a viscous liquid. J Fluid Mech 673:406-431

Harper J (1970) On bubbles rising in line at large Reynolds numbers. J Fluid Mech 41(4):751-758

Howe M (1995) On the force and moment on a body in an incompressible fluid, with application to rigid bodies and bubbles at high and low Reynolds numbers. Q J Mech Appl Math 48(3):401-426

Hurvich CM, Simonoff JS, Tsai CL (1998) Smoothing parameter selection in nonparametric regression using an improved akaike information criterion. J R Stat Soc Ser B (Stat Methodol) 60(2):271-293

Hutchinson M (1986) Algorithm 642: a fast procedure for calculating minimum cross-validation cubic smoothing splines. ACM Trans Math Softw 12(2):150-153

Jeong H, Park H (2015) Near-wall rising behaviour of a deformable bubble at high Reynolds number. J Fluid Mech 771:564-594

Katz J, Meneveau C (1996) Wake-induced relative motion of bubbles rising in line. Int J Multiph Flow 22(2):239-258

Kok JB (1993) Dynamics of a pair of gas bubbles moving trough liquid, part II experiment. Eur J Mech B Fluids 12(4):541-560

Kong G, Mirsandi H, Buist KA, Peters EAJF, Baltussen M, Kuipers JAM (2019) Oscillation dynamics of a bubble rising in a viscous liquid. Exp Fluids 60(8):130

Krakauer NY, Krakauer JC (2012) A new body shape index predicts mortality hazard independently of body mass index. PLoS One 7(7):e39504

Lamb H (1993) Hydrodynamics. Cambridge University Press, Cambridge

Legendre D, Magnaudet J, Mougin G (2003) Hydrodynamic interactions between two spherical bubbles rising side by side in a viscous liquid. J Fluid Mech 497:133-166

Loisy A, Naso A, Spelt PD (2017) Buoyancy-driven bubbly flows: ordered and free rise at small and intermediate volume fraction. J Fluid Mech 816:94-141

Magnaudet J (2015) Lecture notes in hydrodynamics forces acting on gas bubbles

Magnaudet J, Eames I (2000) The motion of high-reynolds-number bubbles in inhomogeneous flows. Ann Rev Fluid Mech 32(1):659-708

Magnaudet J, Mougin G (2007) Wake instability of a fixed spheroidal bubble. J Fluid Mech 572:311-337

Martínez-Mercado J, Palacios-Morales CA, Zenit R (2007) Measurement of pseudoturbulence intensity in monodispersed bubbly liquids for $10<$ re $<500$. Phys Fluids 19(10): 103302

Mirsandi H, Rajkotwala AH, Baltussen MW, Peters EAJF, Kuipers JAM (2018) Numerical simulation of bubble formation with a 
moving contact line using local front reconstruction method. Chem Eng Sci 187:415-431

Mougin G, Magnaudet J (2001) Path instability of a rising bubble. Phys Rev Lett 88(1):014502

Mougin G, Magnaudet J (2002) The generalized Kirchhoff equations and their application to the interaction between a rigid body and an arbitrary time-dependent viscous flow. Int J Multiph Flow 28(11):1837-1851

Newman JN (1977) Marine hydrodynamics. Massachusetts Institute of Technology, Cambridge

Reinsch CH (1967) Smoothing by spline functions. Numerische Math 10(3):177-183

Riboux G, Risso F, Legendre D (2010) Experimental characterization of the agitation generated by bubbles rising at high Reynolds number. J Fluid Mech 643:509-539

Risso F (2018) Agitation, mixing, and transfers induced by bubbles. Ann Rev Fluid Mech 50:25-48

Risso F, Ellingsen K (2002) Velocity fluctuations in a homogeneous dilute dispersion of high-Reynolds-number rising bubbles. J Fluid Mech 453:395-410

Roghair I, Lau Y, Deen N, Slagter H, Baltussen M, Annaland MVS, Kuipers J (2011a) On the drag force of bubbles in bubble swarms at intermediate and high Reynolds numbers. Chem Eng Sci 66(14):3204-3211

Roghair I, Mercado JM, Annaland MVS, Kuipers J, Sun C, Lohse D (2011b) Energy spectra and bubble velocity distributions in pseudo-turbulence: numerical simulations vs. experiments. Int J Multiph Flow 37(9):1093-1098

Roghair I, Baltussen M, Annaland MVS, Kuipers J (2013a) Direct numerical simulations of the drag force of bi-disperse bubble swarms. Chem Eng Sci 95:48-53

Roghair I, Van Sint AM, Kuipers J (2013b) Drag force and clustering in bubble swarms. AIChE J 59(5):1791-1800

Roig V, Roudet M, Risso F, Billet AM (2012) Dynamics of a highReynolds-number bubble rising within a thin gap. J Fluid Mech 707:444-466

Sanada T, Watanabe M, Fukano T, Kariyasaki A (2005) Behavior of a single coherent gas bubble chain and surrounding liquid jet flow structure. Chem Eng Sci 60(17):4886-4900

Sanada T, Sato A, Shirota M, Watanabe M (2009) Motion and coalescence of a pair of bubbles rising side by side. Chem Eng Sci 64(11):2659-2671
Sangani AS, Didwania A (1993) Dynamic simulations of flows of bubbly liquids at large Reynolds numbers. J Fluid Mech 250:307-337

Shew WL, Pinton JF (2006) Dynamical model of bubble path instability. Phys Rev Lett 97(14):144508

Shew WL, Poncet S, Pinton JF (2006) Force measurements on rising bubbles. J Fluid Mech 569:51-60

Smereka P (1993) On the motion of bubbles in a periodic box. J Fluid Mech 254:79-112

Sone D, Sakakibara K, Yamada M, Sanada T, Saito T (2008) Bubble motion and its surrounding liquid motion through the collision of a pair of bubbles. J Power Energy Syst 2(1):306-317

Tripathi MK, Premlata A, Sahu KC, Govindarajan R (2017) Two initially spherical bubbles rising in quiescent liquid. Phys Rev Fluids 2(7):073601

Van Wijngaarden L (1993) The mean rise velocity of pairwise-interacting bubbles in liquid. J Fluid Mech 251:55-78

Van Wijngaarden L (2005) Bubble velocities induced by trailing vortices behind neighbours. J Fluid Mech 541:203-229

Van Wijngaarden L, Jeffrey D (1976) Hydrodynamic interaction between gas bubbles in liquid. J Fluid Mech 77(1):27-44

Vasseur P, Cox R (1977) The lateral migration of spherical particles sedimenting in a stagnant bounded fluid. J Fluid Mech 80(3):561-591

Wahba G (1983) Bayesian "confidence intervals" for the crossvalidated smoothing spline. J R Stat Soc Ser B (Methodol) 45:133-150

Yuan H, Prosperetti A (1994) On the in-line motion of two spherical bubbles in a viscous fluid. J Fluid Mech 278:325-349

Yurkovetsky Y, Brady JF (1996) Statistical mechanics of bubbly liquids. Phys Fluids 8(4):881-895

Zhang J, Chen L, Ni MJ (2019) Vortex interactions between a pair of bubbles rising side by side in ordinary viscous liquids. Phys Rev Fluids 4(4):043604

Publisher's Note Springer Nature remains neutral with regard to jurisdictional claims in published maps and institutional affiliations. 